\title{
TALLYSOMYCIN, A NEW ANTITUMOR ANTIBIOTIC COMPLEX RELATED TO BLEOMYCIN

\author{
III. ANTITUMOR ACTIVITY OF TALLYSOMYCINS A AND B
}

\author{
Hideyo Imanishi, Masaru Ohbayashi, Yūji Nishiyama \\ and Hiroshi KaWAGUCHI \\ Bristol-Banyu Research Institute, Ltd., Meguro, Tokyo, Japan
}

(Received for publication April 17, 1978)

\begin{abstract}
The antitumor activity of tallysomycins A and B was determined in five experimental tumor systems in mice. Tallysomycins A and B were highly active against B16 melanoma, sarcoma 180 ascites tumor and LEWIS lung carcinoma, and moderately active against P388 leukemia but were without effect on lymphoid leukemia L1210. The antitumor activity of tallysomycin A was $2 \sim 3$ times that of tallysomycin B and 3 17 times that of bleomycin. Tallysomycin A was about 1.5 and 4 times more toxic for mice than tallysomycin B and bleomycin, respectively, in terms of subacute $\mathrm{LD}_{50}$ values.
\end{abstract}

Tallysomycin is a new glycopeptide antibiotic complex produced by an unusual actinomycetes strain No. E465-94. Major components A and B of the tallysomycin complex were isolated and characterized, and their antimicrobial properties have been reported ${ }^{1)}$. The structures of tallysomycins $\mathrm{A}$ and B (Fig. 1) have been determined; they are closely related to bleomycin (Fig. 2), differing only in the amino acid composition and by the fact that they possess an additional unique sugar moiety, 4-amino4,6-dideoxy-L-talose ${ }^{2)}$. This paper reports the antitumor activity of tallysomycins A and B in five experimental tumor systems.

\section{Materials and Methods}

Antibiotics:

Copper-free preparations of tallysomycins A and B were used throughout the present study. They were prepared from the copper-chelated form of tallysomycins A and B by the method described in a previous report ${ }^{1)}$. Copper-free preparations of bleomycin complex (consisting mainly of bleomycin $\left.\mathrm{A}_{2}\right)^{3}$ ) and bleomycin $\mathrm{A}_{5}^{4}$ ) which were supplied by Bristol Laboratories, Syracuse, New York, were used as reference agents. Bleomycin $\mathrm{A}_{5}$ (Fig. 2) was included because of its structural similarity to tallysomycin B but the amount available was only enough to test against B16 melanoma. All materials were dissolved in sterile $0.9 \%$ saline and administered to experimental animals intraperitoneally or intravenously.

Animals:

Female $\mathrm{BDF}_{1}(\mathrm{C} 57 \mathrm{BL} / 6 \times \mathrm{DBA} / 2)$ and male dd-strain mice weighing $18 \sim 22 \mathrm{~g}$ were used for the experiments.

Tumors:

Melanotic melanoma B16 was implanted subcutaneously into BDF $_{1}$ mice at an inoculum size of $5 \times 10^{5}$ cells per mouse. Sarcoma 180 ascites tumor was inoculated intraperitoneally into dd mice with $2.5 \times 10^{6}$ cells per mouse. LEWIs lung carcinoma, lymphocytic leukemia P388 and lymphoid leukemia L1210 were implanted intraperitoneally into $\mathrm{BDF}_{1}$ mice using an inoculum of $5 \times 10^{5}, 3 \times 10^{5}$ and $10^{6}$ cells per mouse, respectively.

Treatment:

Twenty-four hours after the implantation of tumor cells, graded doses of test compounds were 
Fig. 1. Structures of tallysomycins A and B

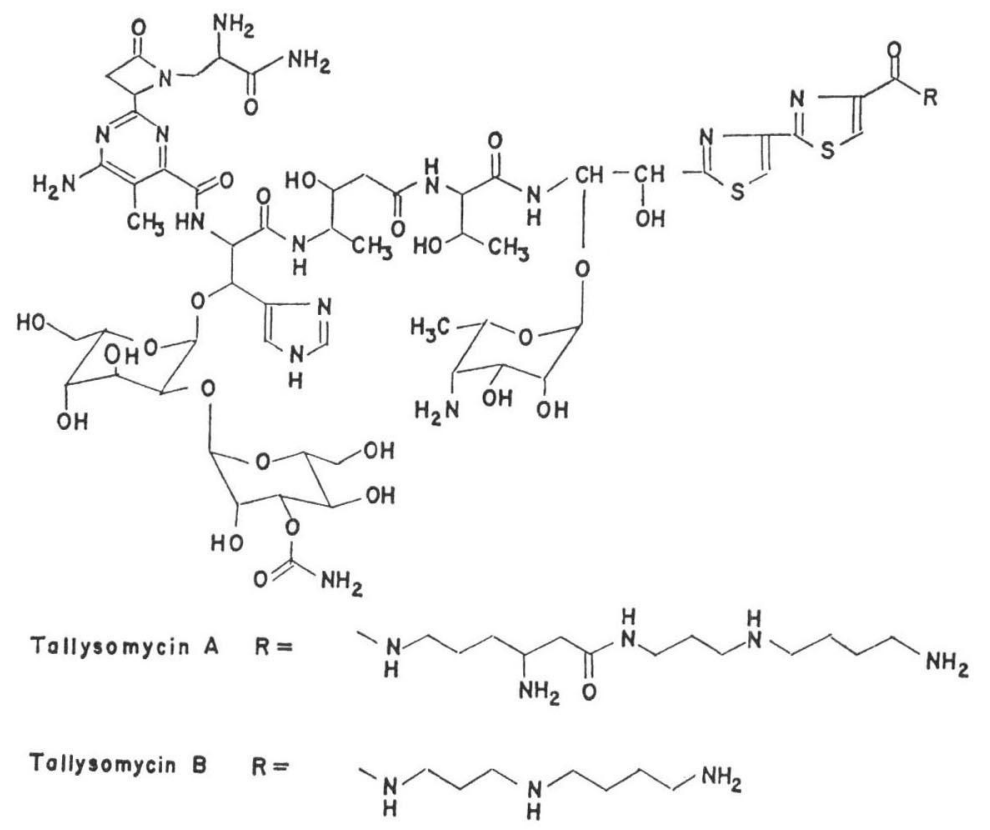

Fig. 2. Structures of bleomycins $\mathrm{A}_{2}$ and $\mathrm{A}_{5}$

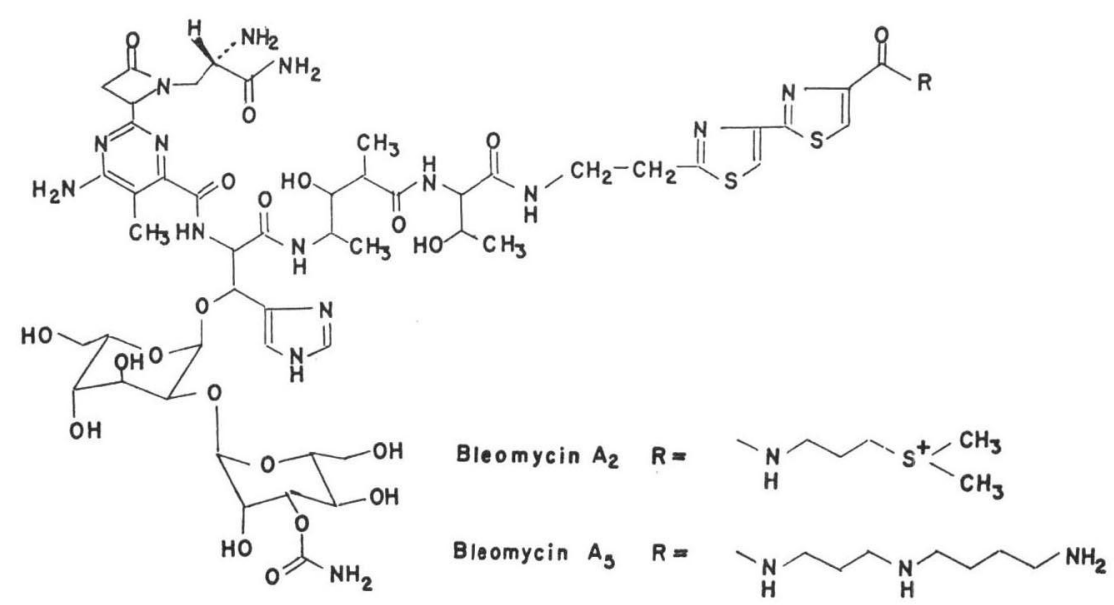

administered to mice intraperitoneally in an injection volume of $0.02 \mathrm{ml}$ per gram of body weight. A logarithmic dilution series consisting of one-half log unit increments of the antibiotics was used for the dose-response study. Treatments were given once daily for 9 days ( $q d 1 \rightarrow 9$ schedule) except for the mice inoculated with Lewis lung carcinoma which were treated for 11 days ( $q d 1 \rightarrow 11$ ).

Evaluation of antitumor effects:

Death or survival of the treated and non-treated (control) animals was recorded daily during the observation period of 45 days after the implantation of tumor cells, and the median survival time (MST) was calculated for each of the test $(\mathrm{T})$ and control (C) groups. A T/C value equal to or greater than $125 \%$ indicates that a significant antitumor effect was achieved. The actual dose giving a T/C of $125 \%$ was estimated by linear regression analysis ${ }^{5)}$ and defined as the effective dose ${ }_{125}$ or $\mathrm{ED}_{125}$. 
In the B16 melanoma experiment the tumor size (long axis $\times$ short axis $\times$ thickness) was measured on day 16 after subcutaneous tumor inoculation, and the dose giving $50 \%$ inhibition of tumor growth $\left(\mathrm{ID}_{50}\right)$ when compared with that of control group was calculated from regression lines.

Toxicity determination:

Graded doses of test compounds were administered to groups of $8 \sim 12$ dd mice intraperitoneally or intravenously. The injection was given one time only or once daily for 9 consecutive days in a volume of $0.02 \mathrm{ml}$ per gram of body weight. Death or survival of the animals was recorded daily for 30 days after the last dose of test compound, and the $\mathbf{L D}_{50}$ was calculated according to the method of VAN DER WAERDEN ${ }^{6}$.

\section{Results}

\section{Melanotic Melanoma B16}

The antitumor activities of tallysomycin A, tallysomycin B, bleomycin complex and bleomycin $\mathrm{A}_{5}$ determined in the B16 melanoma system are shown in Table 1. Tallysomycins A and B showed a doserelated inhibition of tumor growth, the $\mathrm{ID}_{50}$ being $0.26 \mathrm{mg} / \mathrm{kg} /$ day for tallysomycin $\mathrm{A}$ and $0.46 \mathrm{mg} / \mathrm{kg} /$ day for tallysomycin B. The mice treated with tallysomycin also showed a significant prolongation of life span; the increase of MST was more apparent in animal groups in which tumor growth was inhibited greater than $50 \%$. The highest $\mathrm{T} / \mathrm{C}$ value obtained with tallysomycin $\mathrm{A}$ was $183 \%$ at a dose of $1 \mathrm{mg} /$

Table 1. Effects of tallysomycins A and B on B16 melanoma

\begin{tabular}{|c|c|c|c|c|c|c|c|c|}
\hline & \multirow{2}{*}{$\begin{array}{c}\text { Dose } \\
(\mathrm{mg} / \mathrm{kg} / \\
\text { day) }\end{array}$} & \multicolumn{3}{|c|}{ Tumor size at day 16} & \multicolumn{3}{|c|}{ Survival time } & \multirow{2}{*}{$\begin{array}{l}\text { No. survivors } \\
\text { at day } 45 / \\
\text { tested }\end{array}$} \\
\hline & & $\underset{\left(\mathrm{cm}^{3}\right)}{\operatorname{Mean} \pm \text { S.E. }}$ & $\begin{array}{c}\text { Inhibi- } \\
\text { tion } \\
(\%)\end{array}$ & $\begin{array}{c}\mathrm{ID}_{50} \\
\text { (mg/kg/ } \\
\text { day) }\end{array}$ & $\underset{\text { (days) }}{\text { MST/range }}$ & $\begin{array}{l}\mathrm{T} / \mathrm{C} \\
(\%)\end{array}$ & \begin{tabular}{|c}
$\mathrm{ED}_{125}$ \\
$(\mathrm{mg} / \mathrm{kg} /$ \\
day)
\end{tabular} & \\
\hline Control & - & $5.81 \pm 0.47$ & - & - & $23.0 / 21 \sim 43$ & - & - & $0 / 20$ \\
\hline Tallysomycin A & $\begin{array}{l}3 \\
1 \\
0.3 \\
0.1 \\
0.03\end{array}$ & $\begin{array}{l}0.15 \pm 0.08 \\
1.26 \pm 0.24 \\
2.78 \pm 0.39 \\
4.02 \pm 0.65 \\
4.84 \pm 0.75\end{array}$ & $\begin{array}{l}97 \\
78 \\
52 \\
31 \\
17\end{array}$ & 0.26 & $\begin{array}{l}36.5 / 22 \sim 38 \\
42.0 / 26 \sim 43 \\
37.0 / 19 \sim 39 \\
32.0 / 22 \sim 42 \\
27.5 / 16 \sim 42\end{array}$ & $\begin{array}{l}159 \\
183 \\
161 \\
139 \\
120\end{array}$ & 0.042 & $\begin{array}{l}3 / 10^{*} \\
2 / 10 \\
2 / 10 \\
1 / 10 \\
0 / 10\end{array}$ \\
\hline Tallysomycin B & $\begin{array}{l}3 \\
1 \\
0.3 \\
0.1 \\
0.03\end{array}$ & $\begin{array}{l}0.98 \pm 0.21 \\
1.92 \pm 0.18 \\
3.41 \pm 0.49 \\
5.88 \pm 0.49 \\
6.40 \pm 0.91\end{array}$ & $\begin{array}{r}83 \\
67 \\
41 \\
-1 \\
-10\end{array}$ & 0.46 & \begin{tabular}{|c|}
$>45.0 / 31 \sim 38$ \\
$36.5 / 36 \sim 41$ \\
$29.0 / 22 \sim 44$ \\
$29.5 / 21 \sim 42$ \\
$25.0 / 16 \sim 42$
\end{tabular} & \begin{tabular}{|r|}
$>196$ \\
159 \\
126 \\
128 \\
109
\end{tabular} & 0.11 & $\begin{array}{l}7 / 10 \\
1 / 10 \\
0 / 10 \\
0 / 10 \\
0 / 10\end{array}$ \\
\hline $\begin{array}{l}\text { Bleomycin } \\
\text { complex }\end{array}$ & $\begin{array}{c}10 \\
3 \\
1 \\
0.3 \\
0.1\end{array}$ & $\begin{array}{l}0.66 \pm 0.14 \\
1.79 \pm 0.37 \\
2.34 \pm 0.50 \\
4.28 \pm 0.51 \\
6.06 \pm 0.77\end{array}$ & $\begin{array}{r}89 \\
69 \\
60 \\
26 \\
-4\end{array}$ & 0.91 & $\begin{array}{l}37.5 / 14 \sim 45 \\
37.0 / 19 \sim 39 \\
33.0 / 27 \sim 39 \\
30.5 / 24 \sim 43 \\
24.0 / 18 \sim 36\end{array}$ & $\begin{array}{l}163 \\
161 \\
143 \\
133 \\
104\end{array}$ & 0.28 & $\begin{array}{l}0 / 10 \\
0 / 10 \\
2 / 10 \\
0 / 10 \\
0 / 10\end{array}$ \\
\hline Bleomycin $A_{5}$ & $\begin{array}{l}10 \\
3 \\
1 \\
0.3 \\
0.1\end{array}$ & $\begin{array}{l}0.72 \pm 0.10 \\
2.06 \pm 0.09 \\
2.89 \pm 0.59 \\
4.69 \pm 0.65 \\
5.86 \pm 1.33\end{array}$ & $\begin{array}{r}88 \\
65 \\
50 \\
19 \\
-1\end{array}$ & 1.3 & \begin{tabular}{|r|}
$>45.0 / 43 \sim 45$ \\
$35.5 / 31 \sim 41$ \\
$31.0 / 20 \sim 39$ \\
$28.5 / 21 \sim 31$ \\
$20.5 / 20 \sim 40$
\end{tabular} & \begin{tabular}{|r|}
$>196$ \\
154 \\
135 \\
124 \\
89
\end{tabular} & 0.45 & $\begin{array}{l}2 / 4 \\
0 / 4 \\
0 / 4 \\
0 / 4 \\
0 / 4\end{array}$ \\
\hline
\end{tabular}

* 3 of 10 animals died due to toxicity. 
$\mathrm{kg} /$ day. With tallysomycin B the T/C was $>196 \%$ at $3 \mathrm{mg} / \mathrm{kg} /$ day with $7 / 10$ mice still surviving at the termination of the 45 -day observation period. The $\mathrm{ED}_{125}$ of tallysomycins $\mathrm{A}$ and $\mathrm{B}$ in this tumor system was 0.042 and $0.11 \mathrm{mg} / \mathrm{kg} / \mathrm{day}$, respectively. Two reference compounds, bleomycin complex and bleomycin $\mathrm{A}_{5}$, were also active against B16 melanoma, showing $\mathrm{ID}_{50}$ values of 0.91 and $1.3 \mathrm{mg} / \mathrm{kg} / \mathrm{day}$ and $\mathrm{ED}_{125}$ values of 0.28 and $0.45 \mathrm{mg} / \mathrm{kg} / \mathrm{day}$, respectively. These values indicate that tallysomycins $\mathrm{A}$ and B are approximately 7 and 2.5 times as potent as bleomycin complex, respectively, in this tumor system.

\section{Sarcoma 180 Ascites Tumor}

As shown in Table 2, sarcoma 180 was very sensitive to tallysomycins $\mathrm{A}$ and $\mathrm{B}$, the $\mathrm{ED}_{125}$ being 0.02 and $0.042 \mathrm{mg} / \mathrm{kg} /$ day. The animals receiving $0.3 \mathrm{mg} / \mathrm{kg} /$ day of tallysomycin A or $1 \mathrm{mg} / \mathrm{kg} /$ day of tallysomycin B showed the greatest T/C value ( $>293 \%$ for tallysomycin A and $>318 \%$ for tallysomycin B), with one half of the animals receiving these doses still surviving after 45 days. Tallysomycin A was approximately twice as active as tallysomycin B which in turn was about 8 times more potent than bleomycin in terms of $\mathrm{ED}_{125}$ values.

Table 2. Effects of tallysomycins A and B on sarcoma 180 ascites tumor

\begin{tabular}{l|c|c|c|c|c}
\hline & $\begin{array}{c}\text { Dose } \\
(\mathrm{mg} / \mathrm{kg} / \text { day }\end{array}$ & $\begin{array}{c}\text { MST/range } \\
\text { (days) }\end{array}$ & $\begin{array}{c}\mathrm{T} / \mathrm{C} \\
(\%)\end{array}$ & $\begin{array}{c}\mathrm{ED}_{125} \\
(\mathrm{mg} / \mathrm{kg} / \text { day })\end{array}$ & $\begin{array}{c}\text { No. survivors } \\
\text { at day } 45 / \text { tested }\end{array}$ \\
\hline Control & - & $14.0 / 10 \sim 23$ & - & - & $1 / 20$ \\
\hline Tallysomycin A & 3 & $19.0 / 11 \sim 21$ & 136 & 0.020 & $0 / 6$ \\
& 1 & $23.0 / 14 \sim 37$ & 164 & & $3 / 12$ \\
& 0.3 & $>41.0 / 24 \sim 37$ & $>293$ & & $6 / 12$ \\
& 0.1 & $24.0 / 12 \sim 25$ & 171 & & $4 / 12$ \\
& 0.03 & $17.5 / 13 \sim 25$ & 125 & & $4 / 12$ \\
& 0.01 & $16.0 / 7 \sim 24$ & 114 & & $2 / 12$ \\
\hline Tallysomycin B & 3 & $23.0 / 19 \sim 26$ & 164 & 0.042 & $0 / 6$ \\
& 1 & $>44.5 / 20 \sim 44$ & $>318$ & & $6 / 12$ \\
& 0.3 & $36.0 / 12 \sim 45$ & 257 & & $5 / 12$ \\
& 0.1 & $19.5 / 13 \sim 20$ & 139 & & $5 / 12$ \\
& 0.03 & $17.5 / 11 \sim 23$ & 125 & & $2 / 12$ \\
\hline Bleomycin complex & 3 & $27.0 / 16 \sim 27$ & 193 & 0.33 & $5 / 12$ \\
& 1 & $19.0 / 16 \sim 20$ & 136 & & $5 / 12$ \\
& 0.3 & $18.5 / 11 \sim 39$ & 132 & & $4 / 12$ \\
& 0.1 & $13.0 / 10 \sim 25$ & 93 & & $1 / 12$ \\
\hline
\end{tabular}

\section{LewIs Lung Carcinoma}

Table 3 shows the effects of tallysomycins A and B and bleomycin complex on LEwIs lung carcinoma. Significant prolongation of MST was noted in groups of animals receiving tallysomycin A at a daily dose of $0.1 \sim 3 \mathrm{mg} / \mathrm{kg} /$ day, the $\mathrm{ED}_{125}$ being $0.068 \mathrm{mg} / \mathrm{kg} /$ day. Although tallysomycin $\mathrm{B}$ was about one-half as active as tallysomycin $\mathrm{A}$ in terms of the $\mathrm{ED}_{125}(0.16 \mathrm{mg} / \mathrm{kg} /$ day for tallysomycin $\mathrm{B})$, it showed a greater $\mathrm{T} / \mathrm{C}$ value at $3 \mathrm{mg} / \mathrm{kg} /$ day $(215 \%)$ than was achieved with any dosage of tallysomycin A. Bleomycin also showed significant prolongation of MST in the dose range of $0.3 \sim 3 \mathrm{mg} / \mathrm{kg}$ / day, the $\mathrm{ED}_{125}$ being $0.24 \mathrm{mg} / \mathrm{kg} /$ day. The greatest survival rate $(5 / 16)$ was noted in animals receiving 
Table 3. Effects of tallysomycins A and B on Lewis lung carcinoma

\begin{tabular}{l|c|c|c|c|c}
\hline & $\begin{array}{c}\text { Dose } \\
\text { (mg/kg/day) }\end{array}$ & $\begin{array}{c}\text { MST/range } \\
\text { (days) }\end{array}$ & $\begin{array}{c}\mathrm{T} / \mathrm{C} \\
(\%)\end{array}$ & $\begin{array}{c}\mathrm{ED}_{125} \\
(\mathrm{mg} / \mathrm{kg} / \mathrm{day})\end{array}$ & $\begin{array}{c}\text { No. survivors } \\
\text { at day } 45 / \text { tested }\end{array}$ \\
\hline Control & - & $17.0 / 13 \sim 38$ & - & - & $0 / 32$ \\
\hline Tallysomycin A & 3 & $28.0 / 16 \sim 36$ & 165 & 0.068 & $0 / 11$ \\
& 1 & $27.0 / 23 \sim 39$ & 159 & & $0 / 17$ \\
& 0.3 & $25.0 / 19 \sim 42$ & 147 & & $1 / 17$ \\
& 0.1 & $21.5 / 16 \sim 27$ & 126 & & $2 / 17$ \\
& 0.03 & $19.5 / 17 \sim 35$ & 115 & & $0 / 6$ \\
\hline Tallysomycin B & 3 & $36.5 / 25 \sim 43$ & 215 & 0.16 & $2 / 12$ \\
& 1 & $26.0 / 19 \sim 35$ & 153 & & $1 / 18$ \\
& 0.3 & $25.0 / 15 \sim 34$ & 147 & & $0 / 18$ \\
& 0.1 & $19.5 / 15 \sim 34$ & 115 & & $0 / 6$ \\
\hline Bleomycin complex & 0.03 & $18.5 / 17 \sim 34$ & 109 & & $2 / 16$ \\
& 3 & $34.0 / 21 \sim 42$ & 200 & 0.24 & $5 / 16$ \\
& 1 & $26.5 / 19 \sim 38$ & 156 & & $0 / 16$ \\
& 0.3 & $22.0 / 16 \sim 43$ & 129 & & $0 / 12$ \\
\hline
\end{tabular}

a dose of $1 \mathrm{mg} / \mathrm{kg} /$ day of bleomycin. Tallysomycins A and B were about 3.5 and 1.5 times more active than bleomycin, respectively, in terms of $\mathrm{ED}_{125}$ values.

\section{Lymphocytic Leukemia P388}

Table 4 shows the antitumor activity of tallysomycins A and B and bleomycin complex against P388 leukemia. Significant prolongation of life span was seen with doses in the range of $0.3 \sim 3 \mathrm{mg} / \mathrm{kg} /$ day of tallysomycin A, $1 \sim 3 \mathrm{mg} / \mathrm{kg} /$ day of tallysomycin B or $3 \sim 10 \mathrm{mg} / \mathrm{kg} /$ day of bleomycin. The

Table 4. Effects of tallysomycins A and B on P388 leukemia

\begin{tabular}{l|c|c|c|c|c}
\hline & $\begin{array}{c}\text { Dose } \\
(\mathrm{mg} / \mathrm{kg} / \text { day })\end{array}$ & $\begin{array}{c}\text { MST/range } \\
\text { (days) }\end{array}$ & $\begin{array}{c}\mathrm{T} / \mathrm{C} \\
(\%)\end{array}$ & $\begin{array}{c}\mathrm{ED}_{125} \\
(\mathrm{mg} / \mathrm{kg} / \text { day) }\end{array}$ & $\begin{array}{c}\text { No. survivors } \\
\text { at day 45/tested }\end{array}$ \\
\hline Control & - & $9.5 / 7 \sim 13$ & - & - & $0 / 16$ \\
\hline Tallysomycin A & 3 & $14.0 / 11 \sim 15$ & 147 & 0.27 & $0 / 6$ \\
& 1 & $13.0 / 11 \sim 20$ & 137 & & $0 / 12$ \\
& 0.3 & $12.0 / 11 \sim 16$ & 126 & & $0 / 12$ \\
& 0.1 & $11.0 / 8 \sim 16$ & 116 & & $0 / 12$ \\
& 0.03 & $11.0 / 8 \sim 15$ & 116 & & $0 / 12$ \\
\hline Tallysomycin B & 3 & $12.0 / 11 \sim 14$ & 126 & 0.89 & $0 / 6$ \\
& 1 & $12.0 / 10 \sim 18$ & 126 & & $0 / 12$ \\
& 0.3 & $11.0 / 10 \sim 18$ & 116 & & $0 / 12$ \\
& 0.1 & $11.0 / 9 \sim 13$ & 116 & & $0 / 12$ \\
& 0.03 & $10.0 / 7 \sim 13$ & 105 & & $0 / 12$ \\
\hline Bleomycin complex & 10 & $12.5 / 10 \sim 16$ & 132 & 3.3 & $0 / 12$ \\
& 3 & $12.0 / 10 \sim 15$ & 126 & & $0 / 12$ \\
& 1 & $11.0 / 9 \sim 15$ & 116 & & $0 / 12$ \\
& 0.3 & $10.5 / 10 \sim 13$ & 111 & & $0 / 12$ \\
\hline
\end{tabular}


dose-response curves of the three test compounds in the P388 experiments were rather flat, and the maximum T/C values obtained were only $147 \%$ for tallysomycin A, $126 \%$ for tallysomycin B and $132 \%$ for bleomycin. None of the animals receiving these compounds survived until day 20 . The $\mathrm{ED}_{125}$ values were determined to be $0.27,0.89$ and $3.3 \mathrm{mg} / \mathrm{kg} /$ day for tallysomycin $\mathrm{A}$, tallysomycin B and bleomycin, respectively. This indicates that tallysomycins $\mathrm{A}$ and $\mathrm{B}$ were about 12 and 4 times more potent, respectively, than bleomycin.

\section{Lymphoid Leukemia L1210}

As shown in Table 5, L1210 leukemia was quite insensitive to tallysomycins A and B. The greatest $\mathrm{T} / \mathrm{C}$ values obtained were only $112 \%$ for tallysomycin $\mathrm{A}$ (at $0.3 \mathrm{mg} / \mathrm{kg} /$ day) and $106 \%$ for tallysomycin B (at $1 \sim 10 \mathrm{mg} / \mathrm{kg} / \mathrm{day}$ ). All animals treated with tallysomycins A or B died within 10 days, the longest survival period of control animals. Bleomycin was also ineffective against this tumor.

Table 5. Effects of tallysomycins A and B on L1210 leukemia

\begin{tabular}{l|c|c|c|c|c}
\hline & $\begin{array}{c}\text { Dose } \\
(\mathrm{mg} / \mathrm{kg} / \text { day })\end{array}$ & $\begin{array}{c}\text { MST/range } \\
(\text { days })\end{array}$ & $\begin{array}{c}\mathrm{T} / \mathrm{C} \\
(\%)\end{array}$ & $\begin{array}{c}\mathrm{ED}_{125} \\
(\mathrm{mg} / \mathrm{kg} / \text { day })\end{array}$ & $\begin{array}{c}\text { No. survivors } \\
\text { at day } 45 / \text { tested }\end{array}$ \\
\hline Control & - & $8.5 / 7 \sim 10$ & - & - & $0 / 10$ \\
\hline Tallysomycin A & 3 & $9.0 / 8 \sim 10$ & 106 & $>3$ & $0 / 6$ \\
& 1 & $9.0 / 7 \sim 10$ & 106 & & $0 / 6$ \\
& 0.3 & $9.5 / 7 \sim 10$ & 112 & & $0 / 6$ \\
& 0.1 & $8.0 / 7 \sim 10$ & 94 & & $0 / 6$ \\
\hline Tallysomycin B & 10 & $9.0 / 8 \sim 10$ & 106 & $>10$ & $0 / 6$ \\
& 3 & $9.0 / 9 \sim 10$ & 106 & & $0 / 6$ \\
& 1 & $9.0 / 9$ & 106 & & $0 / 6$ \\
\hline \multirow{2}{*}{ Bleomycin complex } & 0.3 & $8.0 / 7 \sim 9$ & 94 & & $0 / 6$ \\
& 10 & $9.0 / 8 \sim 10$ & 106 & $>10$ & $0 / 6$ \\
& 3 & $7.0 / 7 \sim 10$ & 82 & & $0 / 6$ \\
& 1 & $7.0 / 7 \sim 9$ & 82 & & $0 / 6$ \\
\hline
\end{tabular}

\section{Toxicity}

The acute and subacute toxicities of tallysomycins A, B and bleomycin were determined in mice and the $\mathrm{LD}_{50}$ values obtained are summarized in Table 6. Tallysomycin A was 3.1 and 4.1 times more toxic than bleomycin in single intravenous (iv) and intraperitoneal (ip) administrations, respectively. Tallysomycin A was also more toxic $(\times 1.8 \sim 2.4)$ than tallysomycin B. In the multiple dosing schedule (ip, qd $1 \rightarrow 9$ ), tallysomycin $\mathrm{A}$ was about 1.5 times more toxic than tallysomycin $\mathrm{B}$ which in turn was 2.6 times more toxic than bleomycin.

\section{Discussion}

Among the five experimental tumor systems examined in the present study, tallysomycins A and B were highly active against B16 melanoma, sarcoma 180 ascites tumor and LEWIS lung carcinoma, moderately active against P388 leukemia, but inactive against L1210 leukemia. The tallysomycins have also been reported to be active against WALKER carcinosarcoma 256 in rats $^{7)}$. The antitumor effects of tallysomycins A and B were compared with bleomycin complex and their relative activities are sum- 
Table 6. Comparison of antitumor activity and toxicity

\begin{tabular}{|c|c|c|c|c|}
\hline & \multicolumn{4}{|c|}{$\begin{array}{c}\text { Antitumor activity or acute toxicity in } \mathrm{mg} / \mathrm{kg} / \text { day } \\
\text { (relative activity or toxicity) }\end{array}$} \\
\hline & Tallysomycin A & Tallysomycin B & BLM complex & $\mathrm{BLM} \mathrm{A}_{5}$ \\
\hline B16 melanoma $\left(\mathrm{ID}_{50}\right)$ & $0.26 \quad(3.5)$ & $0.46 \quad(2.0)$ & $0.91(1.0)$ & $1.3(0.70)$ \\
\hline B16 melanoma $\left(\mathrm{ED}_{125}\right)$ & $0.042(6.7)$ & $0.11 \quad(2.5)$ & $0.28(1.0)$ & $0.45(0.62)$ \\
\hline Sarcoma $180\left(E_{125}\right)$ & $0.020(17)$ & $0.042(7.9)$ & $0.33(1.0)$ & 一 \\
\hline LL carcinoma $\left(\mathrm{ED}_{125}\right)$ & $0.068(3.5)$ & $0.16 \quad(1.5)$ & $0.24(1.0)$ & - \\
\hline P388 leukemia $\left(E D_{125}\right)$ & $0.27 \quad(12)$ & $0.89 \quad(3.7)$ & $3.3(1.0)$ & - \\
\hline L1210 leukemia $\left(\mathrm{ED}_{125}\right)$ & $>3 \quad(-)$ & $>10 \quad(-)$ & $>10 \quad(-)$ & - \\
\hline IV LD LD (single dose) & $(3.1)$ & $(1.8)$ & $(1.0)$ & - \\
\hline IP $\mathrm{LD}_{50}$ (single dose) & $(4.1)$ & $(1.7)$ & $(1.0)$ & - \\
\hline IP $\mathrm{LD}_{50}(q d 1 \rightarrow 9)$ & $(4.1)$ & $(2.6)$ & $(1.0)$ & - \\
\hline
\end{tabular}

marized in Table 6. Tallysomycin A was generally $2 \sim 3$ times more potent than tallysomycin $\mathrm{B}$, and $3 \sim 17$ times more potent than bleomycin. Tallysomycin A was about 1.5 and 4 times more toxic than tallysomycin B and bleomycin, respectively, when given in a multiple dosing schedule for 9 days. The therapeutic index was obtained for each compound from the ratio of multiple dose $\mathrm{LD}_{50}$ (ip, $q d$ $1 \rightarrow 9$ ) to the $\mathrm{ED}_{125}$ for each of tumor systems (Table 7). Tallysomycin A showed better therapeutic indices than tallysomycin B in all of the tumor systems tested. Bleomycin complex showed somewhat better therapeutic index than that of tallysomycins $\mathrm{A}$ and $\mathrm{B}$ against LewIS lung carcinoma. Against B16 melanoma, sarcoma 180 and P388 leukemia, the therapeutic indices of tallysomycin A were $2 \sim 4$ times superior to those of bleomycin.

Table 7. Comparison of therapeutic indices

\begin{tabular}{l|r|r|r|r|c}
\hline & \multicolumn{5}{|c}{ Therapeutic indices* } \\
\cline { 2 - 6 } & B16 & S180 & LL & P388 & L1210 \\
\hline Tallysomycin A & 105 & 220 & 65 & 16 & $<1.5$ \\
Tallysomycin B & 62 & 162 & 43 & 7.6 & $<0.68$ \\
Bleomycin complex & 64 & 55 & 75 & 5.5 & $<1.8$ \\
\hline
\end{tabular}

* $\quad$ IP $\mathrm{LD}_{50}(q d 1 \rightarrow 9) / \mathrm{ED}_{125}$

Tallysomycin $\mathrm{B}$ is structurally related to bleomycin $\mathrm{A}_{5}{ }^{4}$, both having the same terminal amine moiety. The antitumor activity of tallysomycin B was compared with bleomycin $\mathbf{A}_{5}$ only in the B16 melanoma system. Tallysomycin B showed $3 \sim 4$ times greater activity than bleomycin $\mathbf{A}_{j}$, suggesting some favorable effect of the presence of the aminodeoxytalose moiety and/or the carbinolamine structure $^{2)}$ in the molecule.

Since tallysomycin A differs structurally from tallysomycin B only in that it contains $\beta$-lysine ${ }^{2)}$, the greater biological activity (antitumor and antibacterial ${ }^{1)}$ ) of tallysomycin A over tallysomycin B suggests an important role for this amino acid.

\section{References}

1) Kawaguchi, H.; H. Tsukiura, K. Tomita, M. Konishi, K. Saito, S. Kobaru, K. Numata, K. Fujisawa, T. Mryaki, M. Hatori \& H. Koshiyama: Tallysomycin, a new antitumor antibiotic complex related to bleomycin. I. Production, isolation and properties. J. Antibiotics 30: 779 788, 1977

2) Konishi, M.; K. Saito, K. Numata, T. Tsuno, K. Asama, H. Tsukiura, T. Naito \& H. Kawaguchi: Tallysomycin, a new antitumor antibiotic complex related to bleomycin. II. Structure determination 
of tallysomycins A and B. J. Antibiotics 30: 789 805, 1977

3) Umezawa, H.; K. Maeda, T. Takeuchi \& Y. OKami: New antibiotics, bleomycin A and B. J. Antibiotics, Ser. A19: $200 \sim 209,1966$

4) Fujir, A.; T. Takita, K. Maeda \& H. Umezawa: Chemistry of bleomycin. XI. The structures of the terminal amines. J. Antibiotics 26: 398 399, 1973

5) Sakuma, A. $(e d)$ : Bioassay-Design and Analysis. pp. $110 \sim 111$, University of Tokyo Press, Tokyo, 1970

6) Segawa, T.: Preparation of dose-response curve and calculation of $\mathrm{ED}_{50}$ and $\mathrm{LD}_{50}$. in Iyakuhin Kenkyūhō (Methods of Drug Research) edited by Y. Ban, H. KitaGawa, K. MiYaGi, M. NaKagaKi, T. OKamoto, S. Okui \& H. TAkagi, pp. 91 108, Asakura-shoten, Tokyo, 1968

7) Bradner, W. T.; H. Imanishi, B. S. Hirth \& I. Wodinsky: Antitumor activity and toxicity of Bu-2231 A, a new bleomycin analogue. Proc. Am. Assoc. Cancer Res. 18: 35, 1977 